\section{Inhaled dry powder mannitol in cystic fibrosis: an efficacy and safety study}

\author{
D. Bilton*, P. Robinson*, P. Cooper ${ }^{\mp}$, C.G. Gallagher ${ }^{+}$, J. Kolbe ${ }^{\S}$, H. Fox ${ }^{\dagger}$, A. Jaques ${ }^{\dagger}$ \\ and B. Charlton ${ }^{f}$ for the CF301 Study Investigators**
}

ABSTRACT: This international phase III study of inhaled dry powder mannitol was a randomised, double-blind, 26-week study, followed by a further 26-week, open-label (OL) extension. 324 cystic fibrosis (CF) patients were randomised, in a 3:2 ratio, to mannitol (400 $\mathrm{mg}$ b.i.d.) and control groups. The primary efficacy end-point was to determine the change in forced expiratory volume in $1 \mathrm{~s}$ (FEV 1 ) over the double-blind phase. Secondary end-points included changes in forced vital capacity and pulmonary exacerbations.

A significant improvement in $\mathrm{FEV} 1$ was seen over 26 weeks $(p<0.001)$ and was apparent by 6 weeks, irrespective of concomitant recombinant human deoxyribonuclease (rhDNase) use. At 26 weeks, there was a significant improvement in FEV 1 of $92.9 \mathrm{~mL}$ for subjects receiving mannitol compared with controls (change from baseline $118.9 \mathrm{~mL}(6.5 \%)$ versus $26.0 \mathrm{~mL}(2.4 \%) ; \mathbf{p}<0.001)$. Improvements in FEV1 were maintained up to $\mathbf{5 2}$ weeks in the OL part of the study. There was a $35.4 \%$ reduction in the incidence of having an exacerbation on mannitol $(p=0.045)$.

The incidence of adverse events (AEs) was similar in both groups, although treatment-related AEs were higher in the mannitol compared with the control group. The most common mannitolrelated AEs were cough, haemoptysis and pharyngolaryngeal pain.

Mannitol showed sustained, clinically meaningful benefit in airway function in CF, irrespective of concomitant rhDNase use. Mannitol appears to have an acceptable safety profile for patients with CF.

KEYWORDS: Airway mucociliary clearance, clinical study, cystic fibrosis, dry powder inhalers, forced expiratory volume in $1 \mathrm{~s}$, mannitol dry powder

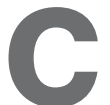
ystic fibrosis (CF) is characterised by a failure to control ion transport across the epithelial cell membrane [1-3]. Several hypotheses have been postulated regarding $\mathrm{CF}$ pathogenesis and the underlying mechanism of CF lung disease. The most widely recognised of these is that in the lungs, there is a relative dehydration and a reduction in volume of airway surface liquid, which is associated with increased mucus viscosity and impaired mucociliary clearance [4-6]. This leads to retention of bacteria and inhaled particles, resulting in chronic airway infection and inflammation, airway damage and respiratory failure $[1,3,7]$. Pulmonary disease is the major cause of morbidity and mortality in $\mathrm{CF}$, with $>90 \%$ of deaths being due to respiratory failure [7]. Pulmonary exacerbations (PEs) are an important clinical feature of $\mathrm{CF}$, as they have both acute and chronic consequences and are the most common reason for hospitalisation, and thus have major influence on healthcare costs [8].

Because CF cannot yet be cured, the goals of therapy are to slow disease progression, alleviate symptoms and improve quality of life (QoL).
Improvement of airway hydration and mucus clearance from the lung is a major therapeutic goal, with the aim of maintaining/restoring respiratory function $[9,10]$. Mannitol is a sugar alcohol that is currently used in medicine as an osmotic agent [11] When inhaled, it creates an osmotic gradient that is thought to facilitate movement of water into the lumen of the airways, thereby increasing the volume of airway surface liquid and improving clearance of mucus $[12,13]$. It is currently used as a bronchial provocation agent in measuring airway hyperresponsiveness [14, 15]. Inhaled mannitol has been shown to benefit patients with bronchiectasis and CF [16-19]. A phase II study demonstrated that inhaled mannitol administered over 2 weeks improved lung function in patients with CF [18]. A dose-finding phase II study further demonstrated that $400 \mathrm{mg}$ b.i.d. inhaled mannitol provided an optimal balance between efficacy, safety and ease of administration [19]. The primary aim of this phase III study was to determine the efficacy and safety of inhaled dry powder mannitol over 26 weeks, with further assessments at week 52 in the open-label (OL) phase of the study.
AFFILIATIONS

*Royal Brompton Hospital, London, UK.

"Royal Children's Hospital, Melbourne,

"The Children's Hospital at Westmead,

${ }^{f}$ Pharmaxis Ltd, Frenchs Forest, Sydney, Australia.

${ }^{+}$St Vincent's University Hospital, Dublin, Ireland

§University of Auckland, Auckland New Zealand

**For a list of the CF302 Study Investigators and their affiliations, see the Acknowledgements section.

CORRESPONDENCE

D. Bilton

Royal Brompton Hospital

Sydney Street

London

SW3 6NP

UK

E-mail: d.bilton@rbht.nhs.uk

Received:

Dec 052010

Accepted after revision: March 202011

First published online: April 082011 


\section{METHODS}

\section{Subjects}

A total of 389 subjects with confirmed CF were enrolled from sites in Australia, Ireland, New Zealand and the UK. Eligible subjects were aged $\geqslant 6$ yrs and had a baseline forced expiratory volume in $1 \mathrm{~s}$ (FEV1) of $\geqslant 30$ and $<90 \%$ predicted. Exclusion criteria included failing a mannitol tolerance test (MTT) at screening, concurrent use of hypertonic saline or $\beta$-blockers, pregnancy or breastfeeding, and intolerance of $\beta$-agonists. Subjects were permitted to continue using recombinant human deoxyribonuclease (rhDNase) and other standard therapies.

Informed written consent was obtained from all participants (or their parent or guardian). The study was conducted in accordance with the Declaration of Helsinki and the protocol was approved by the institutional review board or research ethics committee at each study centre.

\section{Study design}

The first phase of the study was a multicentre, randomised, controlled, parallel-arm, double-blind phase III clinical trial to determine the efficacy and safety of inhaled dry powder mannitol administered over 26 weeks to subjects with CF. This was followed by an optional 26-week, OL extension of the study during which all subjects received inhaled dry powder mannitol.

Prior to randomisation, all subjects were screened for significant airway hyperresponsiveness to mannitol. Following pre-medication with a bronchodilator, the degree of bronchoconstriction after sequential administrations of incremental doses of mannitol, up to a maximum dose of $395 \mathrm{mg}$, was measured. A negative MTT, allowing inclusion in the study, was defined as a $<20 \%$ fall in FEV1 from baseline (or a $\geqslant 20 \%$ fall in FEV1 at the end of the test that had returned to $<20 \%$ within $15 \mathrm{~min}$ ) and arterial oxygen saturation measured by pulse oximetry of $\geqslant 90 \%$ with mannitol, at a total cumulative dose of $395 \mathrm{mg}$.

324 eligible subjects were randomised, using a 3:2 ratio, to a $400 \mathrm{mg}$ inhaled dry powder mannitol b.i.d. group or matched control group. Unlike the first phase II efficacy study, where a nonrespirable dose of mannitol was given, this study used a subtherapeutic $(50 \mathrm{mg})$ dose of mannitol as the control $[18,19]$. The choice of control for this study was based on the need to maintain blinding, provide an appropriate comparator and comply with scientific advice from regulatory agencies. Randomisation was stratified according to concurrent rhDNase use. Subjects took a dose of the short-acting bronchodilator salbutamol (400 $\mu \mathrm{g})$ 5-15 min before each dose of study medication.

Inhaled mannitol was supplied as 10 capsules (40 mg each) together with an inhaler device (RS01 Monodose Inhaler Model 7; Plastiape, Milan, Italy). Capsules were loaded into the inhaler device, punctured, then mannitol inhaled in a deep, controlled manner, followed by a 5-s breath hold. The process was repeated until the contents of 10 capsules were inhaled. The time taken to inhale from 10 capsules was usually 2-5 min. The inhaler device was replaced after 1 week of use to minimise the need for cleaning or disinfection.

As FEV1 is a recommended variable for assessing the efficacy of CF therapies [9], change in FEV1 over 26 weeks was the primary end-point selected for this trial. Secondary end-points included: the percentage of responders (based on a $100 \mathrm{~mL}$ or $\geqslant 5 \%$ relative change in FEV1, or $\geqslant 5 \%$ absolute change in FEV1 $\%$ pred) at 26 weeks; change in other lung function parameters (forced vital capacity (FVC) and forced expiratory flow at 25$75 \%$ of FVC (FEF25-75\%)); protocol-defined PEs (PDPEs) and clinically defined PEs (including exacerbations not meeting the protocol-defined criteria); rescue antibiotic use (number of agents, course and days of use); and QoL scores (using the ageappropriate Cystic Fibrosis Questionnaire) [20]. The PDPEs used the criteria of FuCHS et al. [21], i.e. they were exacerbations in which subjects were treated with intravenous antibiotics for four or more of the following signs or symptoms: change in sputum production; dyspnoea; new or increased haemoptysis; malaise, fatigue or lethargy; fever $\left(\geqslant 38^{\circ} \mathrm{C}\right)$; anorexia or weight loss; sinus pain or tenderness; change in sinus discharge; FVC or FEV1 decreased by $\geqslant 10 \%$ from previous recorded value; radiographic signs indicative of pulmonary infection; increased cough; or changes in physical examination of the chest. Spirometry measurements were taken to coincide with trough treatment effect of inhaled dry powder mannitol; subjects were required to withhold study medication, long-acting $\beta_{2}$-agonists and combination inhaled corticosteroid/long-acting $\beta_{2}$-agonists for $12 \mathrm{~h}$, and short-acting $\beta_{2}$-agonists for $6 \mathrm{~h}$ prior to spirometry assessment. The OL phase included patient follow-up visits at 38 and 52 weeks to assess lung function, sputum microbiology, and review PEs and safety. Safety was assessed by tracking the number and percentage of adverse events (AEs) as well as monitoring for changes in haematology, liver and renal function, and sputum pathogens. Safety and efficacy end-points were examined in cohorts according to rhDNase use as well as within the total cohort.

Treatment compliance was determined by counting returned unused medication and empty blister packaging. The Cystic Fibrosis Questionnaire Revised (CFQ-R) includes several domains to assess QoL, which were analysed separately. An increased score is associated with an improvement in that domain. A difference of four or more points in the respiratory domain is considered to be clinically meaningful [20, 22]. The minimal clinically important differences of other domains have yet to be established.

The study was $80 \%$ powered (two-tailed significance level of $4.98 \%$; primary end-point) to detect a change in FEV1 from baseline at week 26 of $70 \mathrm{~mL}$ in the total intention to treat (ITT) cohort (consisting of all subjects who were randomised and received at least one dose of study medication) and $85 \mathrm{~mL}(5 \%$ significance; secondary end-point) in the subgroup of subjects taking concomitant rhDNase. The study was not powered to detect a difference in exacerbation rate of $<50 \%$.

\section{Statistical analysis}

For the double-blind phase analyses relating to change from baseline in spirometry values, mixed-effect model repeated measure (MMRM) models were used with: absolute change from baseline in spirometry value as the outcome variable; fixed-effect terms for treatment, rhDNase use, geographical region, sex and time-point; disease severity as characterised by FEV1 \% pred at screening, age, baseline spirometry values as covariates; and subject as a random effect. Treatment-by-time, treatment-by-rhDNase use and treatment-by-time-by-rhDNase 
use were also included in models to estimate changes by time, rhDNase use and week 6-26 data. Subject was fitted as a random effect with first order autoregression in the MMRM analysis overall (week 0-26 and 6-26 data). For rate-based analyses (PEs, hospitalisation and antibiotic use), negative binomial regression models were used with treatment, age, rhDNase use and region included as covariates, and the time on study during the blinded phase used as an offset.

PEs were analysed using Cox proportional hazards regression modelling, with treatment, age, rhDNase use and region included as covariates, from which hazard ratios (HRs) were obtained. In addition, Kaplan-Meier estimates of time to first event were provided.

For responder-based analysis (improvement in FEV1 and improvement in QoL respiratory score), subjects were classified as a responders or nonresponders at week 26. A logistic regression model was fitted to compare the odds of responding to mannitol versus control. Terms used in the model were as per the primary analysis model, with response as the outcome, and treatment, age, sex, rhDNase use, region, disease severity as characterised by FEV1 \% pred at screening and baseline FEV1/QoL respiratory score included as covariates.

For the OL analysis, long-term safety was the primary focus, with FEV1 data summarised descriptively at week 52. No between-treatment-group analysis was undertaken.

\section{RESULTS}

\section{Patient disposition}

The ITT study population consisted of subjects (mannitol: $n=177$; control: $n=118$ ) who tolerated mannitol (fig. 1 ), were randomised to treatment, provided a baseline FEV1 and received at least one dose of study medication. The per protocol population (mannitol: $n=111$; control: $n=89$ ) was defined as all subjects in the ITT population with $\geqslant 60 \%$ treatment compliance and no major protocol violations who provided baseline and at least one FEV1 value after commencement of study treatment. The completer population consisted of those subjects who completed the blinded phase of the study (visit 4) with FEV1 data. Patient disposition is presented in figure 1, and demographics and characteristics at baseline are presented in table 1. The median compliance was $89 \%$ and $91 \%$ for mannitol and control groups, respectively.

$85.9 \%$ of the 198 subjects (112 mannitol and 86 control) who completed the double-blind phase elected to enter the optional 26-week OL phase.

\section{Efficacy}

A statistically significant improvement in FEV1 was apparent within 6 weeks of treatment with mannitol and was maintained throughout the double-blind phase of the study (fig. 2). Overall, the treatment effect of mannitol across the study was statistically superior compared with control $(p<0.001)$. The absolute difference in FEV1 averaged across all post-randomisation visits (weeks 6, 14 and 26) in the double-blind phase of the study for mannitol-treated subjects compared with control was $85.03 \mathrm{~mL}(95 \%$ CI 53.5-116.6 mL; $\mathrm{p}<0.001)$; this treatment effect was similar and statistically significant for both the rhDNase subgroup (85.4 mL, 95\% CI 42.9-127.9 mL) and nonrhDNase subgroup (84.6 mL, 95\% CI 38.2-131.1 mL).



FIGURE 1. CF301 trial profile. MTT: mannitol tolerance test; ITT: intention to treat; PP: per protocol; OL: open label. \#: defined as a test that was stopped prior to either a test failure criterion or a cumulative dose of $395 \mathrm{mg}$ being reached.

At 26 weeks, a $118.9-\mathrm{mL}(6.5 \%)$ increase from baseline in the mannitol group resulted in a statistically significant improvement of $92.9 \mathrm{~mL}(\mathrm{p}<0.001)$ in FEV1 compared with control.

Significantly more mannitol-treated subjects achieved clinically meaningful responses compared with control (OR 1.97, $95 \%$ CI $1.08-3.58$ based on a change in FEV1 of $\geqslant 100 \mathrm{~mL}$; $\mathrm{p}=0.026)$ in the completer population. Similar odds of being a responder were seen regardless of the definition of responder used (OR 2.00, 95\% CI 1.09-3.66 and 2.30, 95\% CI 1.20-4.38 for achieving either a FEV1 increase of $\geqslant 5 \%$ or a FEV1 \% pred increase of $\geqslant 5 \%$, respectively; $p<0.05$ for both).

At the end of the double-blind phase, changes in FVC (fig. 3) were consistent with an improvement in FEV1 (at week 26, FVC increase from baseline was 128.9 versus $15.9 \mathrm{~mL}$ for the mannitol and control groups, respectively; $\mathrm{p}=0.002$ ). FEF25-75\% also improved with mannitol, but compared with control, differences in FEF25-75\% were not statistically significant.

During the uncontrolled, OL phase of the study, subjects who were switched to mannitol after initial randomisation to control in the double-blind phase showed a clinically and statistically 


\section{TABLE 1 Patient demographic data and baseline characteristics}

\begin{tabular}{|c|c|c|c|}
\hline Characteristics & Mannitol & Control & All \\
\hline Subjects & 177 & 118 & 295 \\
\hline $6-11$ & $31(17.5)$ & $17(14.4)$ & $48(16.3)$ \\
\hline $12-17$ & $32(18.1)$ & $25(21.1)$ & $57(19.3)$ \\
\hline$\geqslant 18$ & $114(64.4)$ & $76(64.4)$ & $190(64.4)$ \\
\hline $\mathrm{BMI} \mathbf{k g} \cdot \mathrm{m}^{-2}$ & $21.07 \pm 3.99$ & $20.38 \pm 3.59$ & $20.80 \pm 3.84$ \\
\hline Females & $71(40.1)$ & $61(51.7)$ & $132(44.7)$ \\
\hline FEV $_{1} L_{2}$ & $2.07 \pm 0.82^{\# \#}$ & $1.95 \pm 0.69$ & $2.02 \pm 0.77^{\circ \varphi}$ \\
\hline FEV $_{1} \%$ pred & $62.4 \pm 16.45^{\# \#}$ & $61.4 \pm 16.13$ & $62.0 \pm 16.30^{\circ}$ \\
\hline \multicolumn{4}{|l|}{ Microbiology } \\
\hline \multicolumn{4}{|l|}{ Pseudomonas aeruginosa } \\
\hline Aspergillus spp. & $28(15.8)$ & $11(9.3)$ & 39 (13.2) \\
\hline \multicolumn{4}{|l|}{ Chronic antibiotic use $>10 \%$ at baseline } \\
\hline Azithromycin & $98(55.4)$ & $60(50.8)$ & $158(53.6)$ \\
\hline Colistin $\#$ & $73(41.2)$ & $45(38.1)$ & $118(40.0)$ \\
\hline Tobramycin ${ }^{\#}$ & $43(24.3)$ & $35(29.7)$ & $78(26.4)$ \\
\hline Flucloxacillin & $5(25.4)$ & $23(19.5)$ & $68(23.1)$ \\
\hline Drugs for obstructive airway diseases & $148(83.6)$ & $101(85.6)$ & $249(84.4)$ \\
\hline $\mathrm{ICS}^{\circ}$ & $103(58.2)$ & $73(61.9)$ & $176(59.7)$ \\
\hline $\mathrm{SABA}^{+}$ & $137(77.4)$ & $96(81.4)$ & $233(79.0)$ \\
\hline$\angle A B A^{\S}$ & $98(55.4)$ & $64(54.2)$ & $162(54.9)$ \\
\hline Other ${ }^{f}$ & $38(21.5)$ & $21(17.8)$ & $59(20.7)$ \\
\hline rhDNase users & $96(54.2)$ & $67(56.8)$ & $163(55.3)$ \\
\hline
\end{tabular}

Data are presented as $n$, mean \pm SD or $n(\%)$. BMI: body mass index; FEV1: forced expiratory volume in $1 \mathrm{~s}$; \% pred: \% predicted; ICS: inhaled corticosteroid; SABA: shortacting $\beta$-agonist; LABA: long-acting $\beta$-agonist; rhDNase: recombinant human deoxyribonuclease. ${ }^{\#}$ : $98.3 \%$ of colistin and $92.3 \%$ of tobramycin was nebulised at baseline; $\uparrow$ : including combinations and single agents; ${ }^{+}$: salbutamol and terbutaline; ${ }^{\S}$ : salmeterol, formoterol or combinations; ${ }^{f}$ : including leukotriene receptor antagonists, anticholinergic bronchodilators, theophylline, aminophylline and nedocromil; ${ }^{\# \#}: n=176 ;{ }^{~}{ }^{\mu}: n=294 ;{ }^{++}: n=95$.

significant improvement in FEV1 that was comparable to the improvements seen in the mannitol group during the doubleblind phase of the study. Subjects initially randomised to mannitol maintained the increase in FEV1 seen during the double-blind phase over the second 26 weeks (fig. 4 ). The change in FEV1 in members of the control group who moved to the OL phase, subsequently received mannitol for 6 months and completed the study showed an absolute improvement from baseline $(148.5 \mathrm{~mL})$, which was similar to that seen with the completers from the original mannitol group (155.7 mL).

The PDPE results are presented in table 2. There was a significant $35.4 \% \quad(p=0.045)$ reduction in the incidence of having a PDPE during the double-blind phase of the study, although there was no statistically significant reduction in the rate of exacerbations. For the per protocol patient population, there was a significant increase in the time to first exacerbation (HR 0.47; $p=0.024$ ) (fig. 5), which did not reach statistical significance in the ITT population (HR 0.68; $p=0.119)$.

The trend toward improvement in exacerbation-related endpoints was consistent with lung function improvement, and there was a correlation between PDPE incidence and improvement in FEV1 over the 26 weeks of the study $(p<0.0001)$.

The results from the CFQ-R treatment burden domain at baseline were similar to the results at the end of the study, demonstrating that there was no meaningful increase in treatment burden resulting from additional therapy with 10 capsules in a dry powder inhaler twice a day.

There was a 3.8-point difference in mean change from baseline in the CFQ-R respiratory score in favour of the mannitol group 



FIGURE 2. Change $(\Delta)$ in forced expiratory volume in $1 \mathrm{~s}(\mathrm{FEV} 1)$ over the 26 -week blinded phase in patients in the mannitol and control groups. $\Delta \mathrm{FEV} 1$ in a) the whole intention to treat (ITT) group ( $\Delta \mathrm{FEV} 1118.9 \mathrm{~mL}(6.5 \%)$ for mannitol from baseline to week $26 ; p<0.001$ for mannitol versus control over 26 weeks), b) the subgroup of ITT patients receiving recombinant human deoxyribonuclease (rhDNase) ( $\Delta$ FEV1 $81.0 \mathrm{~mL}(4.6 \%)$ for mannitol from baseline to week 26 ; $p=0.008$ for mannitol versus control over 26 weeks) and c) the non-rhDNase group ( $\Delta \mathrm{FEV} 1158.3 \mathrm{~mL}$ (8.5\%) for mannitol from baseline to week 26; $p=0.015$ for mannitol versus control over 26 weeks), and d) relative percentage change in FEV1 \% predicted in the ITT group ( $\Delta \mathrm{FEV} 14.21 \%$ pred for mannitol from baseline to week $26 ; \mathrm{p}=0.004$ for mannitol versus control over 26 weeks). Data are presented as least-squares mean $\pm \mathrm{SE}$. The mean changes are based on a repeated-measures analysis. A mixed-effects model was used to determine the effects across the 26-week period (weeks 0-26 for a-c and weeks 6-26 for d), with $\Delta F E V_{1}$ as the outcome; treatment, time-point, rhDNase use, geographical region and sex as fixed effects; and baseline FEV1, age and disease severity (baseline FEV1 \% pred) as covariates. A treatment effect by time-point was obtained by adding a time-by-interaction term to the model.

$(\mathrm{p}=0.096)$. Differences in the vitality and physical domains were 7.2 and 4.2 points, respectively, again trending in favour of mannitol.

\section{Safety}

Overall, the proportion of subjects reporting at least one AE during the blinded study period was similar for the mannitol and control groups (any AE: 87.0 versus 92.4\%, respectively; serious AE: 26.0 versus $29.7 \%$, respectively). Pulmonary exacerbations are an expected feature of the underlying disease in $\mathrm{CF}$ and were carefully documented as an efficacy end-point as well as being recorded as AEs (condition aggravated) and this was the most commonly reported $\mathrm{AE}$ in both treatment groups. Treatment-related AEs were reported in $40.7 \%$ of mannitoltreated subjects and $22 \%$ of the control group. AEs that were more common in the mannitol group included cough, haemoptysis (see later) and pharyngolaryngeal pain. Lower respiratory tract infection and exacerbation were less common in the mannitol group than in the control group (table 3).
Haemoptysis was reported either as an AE (11.9\% versus $8.5 \%$ in the mannitol and control groups, respectively) or as a sign in association with an exacerbation; overall, haemoptysis, including that associated with exacerbations of $\mathrm{CF}$, was reported by $15.8 \%$ of mannitol-treated subjects and $15.3 \%$ of subjects receiving the control. The majority of the cases of haemoptysis were considered to be not related to the study drug, transient and mild-to-moderate in intensity.

A total of $28(15.8 \%)$ subjects in the mannitol group and 10 $(8.5 \%)$ in the control group discontinued the study due to AEs; of these, $24(13.6 \%)$ and six (5.1\%) subjects, respectively, withdrew due to treatment-related AEs (table 3). All subjects who withdrew due to a serious $\mathrm{AE}$ were in the mannitol group (moderate haemoptysis: $n=2$; moderate severity PE: $n=1$; severe, asymptomatic bronchoconstriction: $n=1$ ).

The incidence of AEs and serious AEs among rhDNase users and nonusers were similar to the overall results in both treatment groups. More rhDNase users in both treatment 




FIGURE 3. Change $(\Delta)$ in forced vital capacity (FVC) for the 26-week blinded phase in patients receiving mannitol or control in the intention to treat group ( $\Delta \mathrm{FVC}$ $128.9 \mathrm{~mL}$ (4.95\%) for mannitol from baseline to week 26; $p<0.001$ for mannitol versus control over 26 weeks). Data are presented as least-squares mean \pm SE. The mean changes are based on a repeated-measures analysis. A mixed-effects model was used to determine the effects across the 26-week period (week 0-26), with $\triangle \mathrm{FVC}$ as the outcome; treatment, time-point, recombinant human deoxyribonuclease use, geographical region and sex as fixed effects; and baseline forced expiratory volume in $1 \mathrm{~s}\left(\mathrm{FEV} \mathrm{V}_{1}\right.$ ), age and disease severity (baseline $\mathrm{FEV}_{1} \%$ predicted) as covariates. A treatment effect by time-point was obtained by adding a time-by-interaction term to the model.

groups $(32.3 \%$ and $32.8 \%$ subjects in the mannitol and control groups, respectively) experienced serious adverse events than rhDNase nonusers (18.5 and $25.5 \%$ subjects, respectively).

The most common organisms in both treatment groups at week 0 were Pseudomonas aeruginosa (mucoid and nonmucoid), Staphylococcus aureus and Aspergillus spp. At week 26, the proportion of subjects in both treatment groups with abnormal flora was similar to week 0 for all grades of growth. Sputum microbiology was similar for rhDNase users and nonusers, and for paediatric and adolescent subjects. There were no apparent differences in laboratory results for mean haematology, liver function, urea and electrolyte parameters between treatment groups or over time.

\section{DISCUSSION}

Treatment with inhaled dry powder mannitol (400 mg b.i.d.) provided early and sustained, statistically and clinically significant increases in FEV1 over a 26-week treatment period $(\mathrm{p}<0.001)$ in subjects with CF receiving a high standard of care. This improvement was maintained to 52 weeks in the OL phase of the study.

The improvement in lung function compared with control was seen irrespective of concomitant rhDNase use. The additional benefit of mannitol over the study period was very similar in rhDNase users and nonusers when compared with control; this is in contrast with a recent, small phase II study [23], which suggested that the combined use of rhDNase and mannitol may not result in greater improvement than rhDNase alone. The present larger study demonstrated that mannitol offered an additional lung function benefit on top of rhDNase as part of standard therapy and confirms its applicability to a wide range of CF patients.

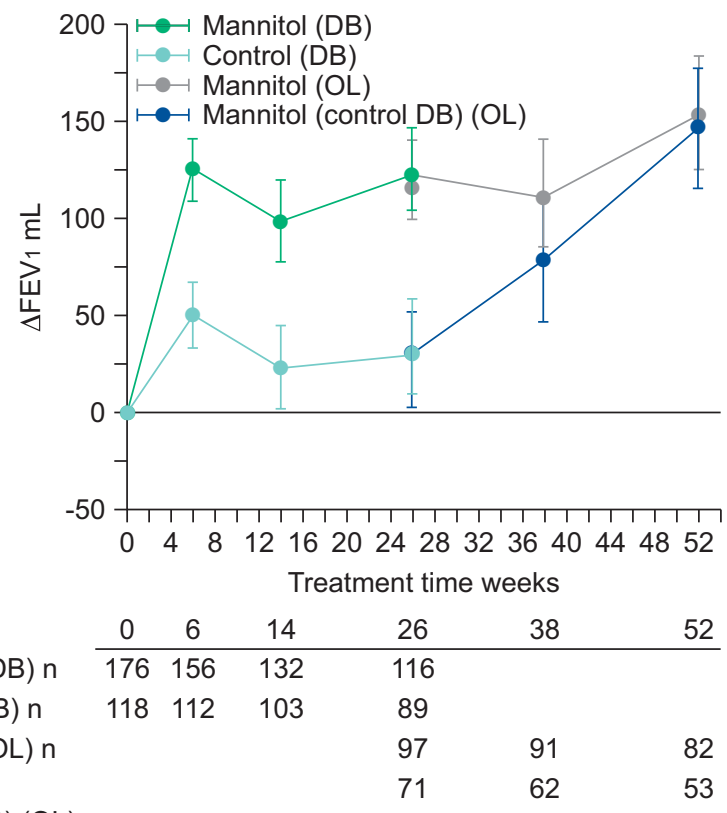

(control DB) (OL) n

FIGURE 4. Change $(\Delta)$ in forced expiratory volume in $1 \mathrm{~s}$ (FEV1) from baseline over the double-blind (DB) (weeks 0-26) and open-label (OL) (weeks 26-52) phases of the study. Data are presented as least squares mean \pm SE. Control patients went on to receive mannitol in the OL phase of the study. Numbers of patients contributing to each mean $\mathrm{FEV}_{1}$ at each time-point are presented below the graph.

The present study also evaluated the proportion of subjects reaching a clinically meaningful threshold change in FEV1. There are no guidelines on this threshold in CF, and the size of effect required to be meaningful varies according to individual perception, pre-existing lung impairment, size and age. For this study, a conservative threshold for a clinically significant improvement from baseline in FEV1 was evaluated by absolute changes of both $100 \mathrm{~mL}$ and 5\% FEV1 \% pred, as well as a 5\% change in FEV1. More subjects treated with mannitol reached these clinically meaningful lung function thresholds. Improvements with mannitol using other lung function parameters (such as the FVC and peak expiratory flow) reflected the positive improvements in FEV1 and support the consistent effect of mannitol on lung function. While FEF25-75\% was not statistically different between the mannitol and control groups, the variability of this measure across the study was high and the mean change from baseline with mannitol at week 26 was $86.2 \mathrm{~mL} \cdot \mathrm{s}^{-1}\left(95 \%\right.$ CI $\left.29.33-143.11 \mathrm{~mL} \cdot \mathrm{s}^{-1}\right)$.

The frequency of PEs is an important outcome measure in clinical trials, as exacerbations contribute to the burden of disease and are associated with impaired QoL, increased hospitalisation and associated healthcare costs, and increased risk of mortality $[8,9,24,25]$. In the present study, many different measures of exacerbation, including incidence and rate of PDPE or clinically defined PE, time to first PDPE or clinically defined PE, and rescue antibiotic use, were assessed in a range of patient populations (ITT population, per protocol population and completers). There was a significant reduction in the incidence of having a PDPE in the ITT group and 
TABLE 2 CF301 protocol-defined pulmonary exacerbation (PDPE) data

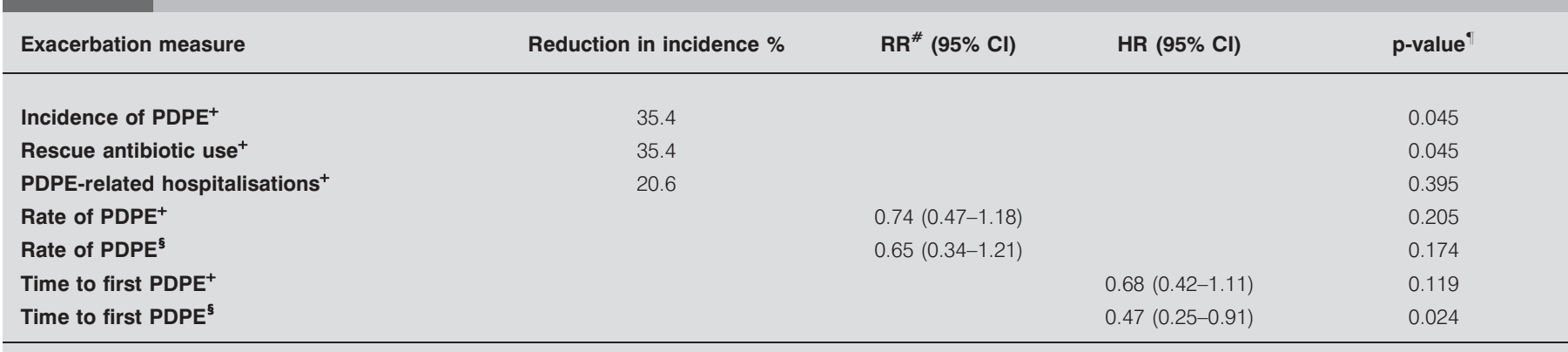

RR: rate ratio; HR: hazard ratio. ${ }^{\#}$ : negative binomial regression of the rate; ${ }^{9}$ : post hoc Chi-squared test for mannitol versus control; ${ }^{+}$: intention to treat; ${ }^{\text {s: }}$ per protocol.

significant improvement in time to first exacerbation in the per protocol population with mannitol $(p=0.024)$, while other exacerbation end-points did not reach statistical significance. Nevertheless, the trial was not powered to detect differences in exacerbation rates that would still be considered clinically meaningful. Currently, only two nebulised agents are used in clinical practice to help airway clearance in CF subjects. Inhaled mannitol demonstrated a similar benefit in terms of relative percentage improvement in lung function to rhDNase $10 \mathrm{yrs}$ in most countries [21, 26]. This similar improvement with mannitol is considered significant given the differing standards of care available at the time of the original rhDNase studies and in a population who are now slightly older and on more concomitant medications (including $\beta$-agonists and antibiotics). Hypertonic saline is frequently used as an osmotic agent at various dosing frequencies, doses and concentrations, although these are largely driven by tolerability. The primary evidence base for hypertonic saline comes from one study in a (a mucolytic), which has had regulatory approval for well over

milder population of 164 patients where $4 \mathrm{~mL}$ of $7 \%$ saline was used twice a day to improve lung function and exacerbation rates [27]. The primary outcome measure of rate of change in lung function was not significant between groups over the duration of the study. By comparison, mannitol improved FEV1 early (at 6 weeks) in this study, and the significant effect size with mannitol was maintained out to 26 weeks and further, to 52 weeks in patients extending the treatment period. Compared with this study using mannitol, the hypertonic saline study by ELKINS et al. [27], with its notably lower rates of regular concomitant antibiotic use, demonstrated a statistically significant reduction in exacerbation rate, although a similar magnitude of reduction in incidence of exacerbations was reported (35\% for mannitol versus 37\% for hypertonic saline).

Osmotic agents offer a rational approach to the treatment of CF and nonionic agents such as mannitol would hypothetically have a more sustained duration of action in the airway, as epithelia may remove salts ions more rapidly [5]. Mannitol has

b)



\begin{tabular}{|cccc|}
\hline & Patients & Events & Censored \\
Control & 118 & 33 & 85 \\
Mannitol & 177 & 32 & 145 \\
\hline
\end{tabular}

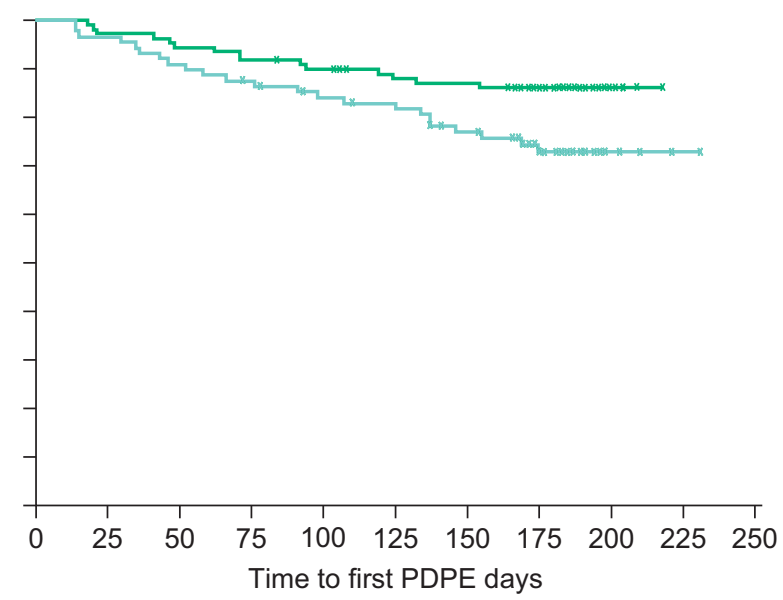

\begin{tabular}{|cccc|}
\hline & Patients & Events & Censored \\
Control & 89 & 23 & 66 \\
Mannitol & 111 & 15 & 96 \\
\hline
\end{tabular}

FIGURE 5. Protocol-defined pulmonary exacerbation (PDPE) event-free survival in the a) intention to treat population (hazard ratio (HR) $0.68,95 \% \mathrm{Cl} 0.42-1.11$; $\mathrm{p}=0.119$ ) and $\mathrm{b}$ ) per protocol population ( $\mathrm{HR} 0.47,95 \% \mathrm{Cl} 0.25-0.91 ; \mathrm{p}=0.024)$. $\times$ : censored subjects (those with no pulmonary exacerbations at the time of their last contact in the blinded study period (either week 26 or early withdrawal)). The study has 2-week windows for each visit, which allowed a number of patients in both arms to continue the double-blinded phase for the study for $\leqslant 8$ months. 


\begin{tabular}{|c|c|c|c|}
\hline At least one AE & $154(87.0)$ & $109(92.4)$ & $263(89.2)$ \\
\hline At least one treatment-related $\mathrm{AE}$ & $72(40.7)$ & $26(22.0)$ & $98(33.2)$ \\
\hline At least one SAE & $46(26.0)$ & $35(29.7)$ & $81(27.5)$ \\
\hline Condition aggravated & 57 (32.2) & $42(35.6)$ & 99 (33.6) \\
\hline Cough & $45(25.4)$ & $24(20.3)$ & $69(23.4)$ \\
\hline Headache & $38(21.5)$ & $28(23.7)$ & $66(22.4)$ \\
\hline Bacteria sputum identified & $33(18.6)$ & $22(18.6)$ & $55(18.6)$ \\
\hline Nasopharyngitis & $25(14.1)$ & $17(14.4)$ & $42(14.2)$ \\
\hline \multicolumn{4}{|c|}{ Treatment-related AEs leading to withdrawal in $\geqslant 2$ subjects } \\
\hline Cough & $11(6.2)$ & $4(3.4)$ & $15(5.1)$ \\
\hline Haemoptysis & $5(2.8)$ & $0(0.0)$ & $5(1.7)$ \\
\hline Condition aggravated & $3(1.7)$ & $1(0.8)$ & $4(1.4)$ \\
\hline Chest discomfort & $3(1.7)$ & $0(0.0)$ & $3(1.0)$ \\
\hline Bronchospasm & $2(1.1)$ & $0(0.0)$ & $2(0.7)$ \\
\hline Pharyngolaryngeal pain & $2(1.1)$ & $0(0.0)$ & $2(0.7)$ \\
\hline Wheezing & $0(0.0)$ & $2(1.7)$ & $2(0.7)$ \\
\hline
\end{tabular}

Data are presented as $n$ or $n(\%)$. SAE: serious AE. * : occurring in $\geqslant 10 \%$ subjects.

properties that make it a good osmotic agent for the treatment of CF: it has sufficient osmotic load per unit weight, can be formulated as a powder for inhalation, is stable and is not subject to ionic movement.

Noncompliance with and adherence to therapy are important issues in $\mathrm{CF}$, and can influence the therapeutic value of treatment. Therapies are often time consuming and challenging to deliver, so adherence may be poor, especially among certain groups, such as adolescents [28, 29]. Dry powder mannitol offers the convenience of delivery to the lung via a simple, hand-held inhaler, rather than via a nebuliser, and it can be administered within 5 min [30]. The shorter delivery time and lack of need for sophisticated equipment, with its associated need for maintenance and cleaning, may lead to improved adherence, although this would need to be confirmed in future studies. The withdrawal rate at the start of this study was higher than expected; however, the need to provide education about the device and expectations for patients and healthcare professionals may have been underestimated. The impact of additional education will be able to be evaluated from the second phase III study, CF302 (ClinicalTrials.gov identifier NCT00630812).

Improvements in QoL scores for vitality, respiratory and physical domains are all considered to be relevant in the assessment of a pulmonary-directed therapy; differences in these scores all approached or exceeded 4 points, but did not reach statistical significance. While the CFQ-R is a widely accepted and validated tool for use in patients with $C F$, questions such as "Have you had to cough up mucus?" and "Have you been coughing during the day?" may favour the control group, as sputum production with mannitol increased during the study compared to control, and thus, the tool may be inappropriate for reflecting improvements associated with mucoactive therapies.

Mannitol was well tolerated by most study subjects with an acceptable safety profile. Cough and haemoptysis were the most commonly reported treatment-related AEs. Haemoptysis, similar to exacerbations, is an underlying feature of CF and, therefore, is expected in CF studies [31]. The total incidence of haemoptysis when occurring either during a PE or as a treatment-related $\mathrm{AE}$ was, however, similar between the two treatment groups. Cough was more common in the mannitol group but, as it may be considered to be a component of the therapeutic effect, it is difficult to interpret the potential benefit/risk ratio of this finding. Importantly, cough was only sufficiently troublesome in a small proportion of subjects to lead to treatment discontinuation. Overall, most AEs were mild or moderate in intensity.

As mannitol may potentially cause bronchoconstriction in those with airway hyperresponsiveness, the trial excluded such subjects at screening with an MTT. However, $87.8 \%$ of screened subjects passed the MTT.

Exploratory analyses of bronchoconstrictor response to study drug at each double-blind visit and occurrence of bronchoconstriction-related AEs were performed. There was 
no suggestion of an increased risk of airway hyperresponsiveness in either the measured response to mannitol or when reviewing the cluster of associated side-effects of wheeze, asthma and bronchospasm. Although there was a greater proportion of subjects in the mannitol group compared with those in the control group who had a medical history of asthma (34.5 versus $19.5 \%$ ), the proportion of subjects experiencing AEs pertaining to bronchospasm was low and comparable between the two treatment groups (bronchospasm: $1.1 \%$ versus $0.0 \%$; asthma: $1.1 \%$ versus $2.5 \%$; dyspnoea: $1.1 \%$ versus $0.8 \%$; wheezing: $2.3 \%$ versus $3.4 \%$ ).

The study results suggest there is no added risk involved in treating subjects with mannitol with respect to sputum growth of microorganisms. Mannitol is a growth substrate for certain bacteria in vitro, particularly S. aureus, Burkholderia cepacia and $P$. aeruginosa [32]. It was therefore important to assess whether this had an impact on sputum microbiology over time. While most subjects grew abnormal flora in their sputum at randomisation, there was no evidence for either an increase or decrease in microorganism growth after 26 weeks of treatment.

In those who completed the double-blind period, the study drug appears to have been well tolerated, as the majority $(85 \%)$ of subjects elected to continue into the OL study.

Consistent with regulatory agency guidance, the control used was an anticipated subtherapeutic 50-mg dose of mannitol, meaning that the size of the effect is possibly conservative, but safety (essentially that related to the inhaled route) when assessed relative to control may potentially be underestimated (although incidence of AE rates did not exceed expectations, based on the incidence in published studies [21, 33]). Nevertheless, while this could be considered a possible limitation to the study, the use of a subtherapeutic mannitol dose was supported by the dose-response study (DPM-CF-202) [26], in which a single 40-mg capsule b.i.d. did not demonstrate any efficacy over a 2-week treatment period, while the 400-mg therapeutic dose was efficacious.

In conclusion, inhaled mannitol appears to have an acceptable safety profile for patients with CF and demonstrates that mannitol treatment provides both early and sustained clinically meaningful improvements in airway function over and above the existing standard care, irrespective of concomitant rhDNase use, in a representative population of CF subjects.

\section{SUPPORT STATEMENT}

This work was supported by the NIHR Respiratory Disease Biomedical Research Unit at the Royal Brompton and Harefield NHS Foundation Trust, and Imperial College London (London, UK). The study received funding from Pharmaxis Ltd (Sydney, Australia). The study sponsor participated in the study design, data collection, data analysis, data interpretation and writing of the report. Following completion of the trial, the data were held and analysed by the sponsor.

\section{CLINICAL TRIAL}

This study is registered at ClinicalTrials.gov with identifier number NCT00446680.

\section{STATEMENT OF INTEREST}

Statements of interest for all authors and for the study itself can be found at www.erj.ersjournals.com/site/misc/statements.xhtml

\section{ACKNOWLEDGEMENTS}

The CF301 Study Investigators were: S. Bell (The Prince Charles Hospital, Brisbane, Australia), A. Chang (Royal Children's Hospital, Brisbane), P. Cooper (The Children's Hospital at Westmead, Sydney, Australia), I. Feather (Gold Coast Hospital, Southport, Australia), H. Greville (Royal Adelaide Hospital, Adelaide, Australia), A. Jaffe (Sydney Children's Hospital, Randwick, Australia), D. Serisier (Mater Adult Hospital, Brisbane), M. Phillips (Sir Charles Gairdner Hospital, Perth, Australia), P. Robinson (Royal Children's Hospital, Melbourne, Australia), A. Young (The Alfred Hospital, Melbourne), G. Canny (Our Lady's Hospital, Dublin, Ireland), C. Gallagher (St Vincent's University Hospital, Dublin), P. Greally (National Children's Hospital, Dublin), N.G. McElvaney (Beaumont Hospital, Dublin), J. Kolbe (Green Lane Clinical Centre, Auckland, New Zealand), R. Taylor (Dunedin Hospital, Dunedin, New Zealand), D. Bilton (Royal Brompton Hospital, London, UK), A. Cade (Plymouth and Derriford Hospital, Plymouth, UK), S. Carr (Barts and the London NHS Trust, London), M. Carroll (Southampton General Hospital, Southampton, UK), M. Desai (Birmingham Children's Hospital, Birmingham, UK), S. Elborn (Belfast City Hospital, Belfast, UK), I. Doull (Children's Hospital for Wales, Cardiff, UK), C. Haworth (Papworth Hospital Cystic Fibrosis Centre, Cambridge, UK), I. Ketchell (Llandough Hospital, Cardiff), A. Knox (Nottingham City Hospital, Nottingham, UK), L. Kuitert (Barts and the London NHS Trust), S. Langton-Hewer (Bristol Royal Hospital for Children, Bristol, UK), J. Legg (Southampton General Hospital), C. Murray (Royal Manchester Children's Hospital, Manchester, UK), N. Jarad (Bristol Royal Infirmary, Bristol), D. Peckham (St James University Hospital, Leeds, UK), S. Range (Glenfield Hospital, Leicester, UK), S. Robertson (Royal Devon and Exeter NHS Foundation, Exeter, UK), A. Smyth (Queens Medical Centre, Nottingham), K. Southern (Alder Hey Children's Hospital, Liverpool, UK), D. Spencer (Royal Victoria Infirmary, Newcastle-upon-Tyne, UK), C. Taylor (Sheffield Children's Hospital, Sheffield, UK), C. Upton (Norfolk and Norwich University Hospital, Norwich, UK), M. Walshaw (Liverpool Heart and Chest Hospital, Liverpool) and J. Whitehouse (Birmingham Heartland Hospital, Birmingham).

D. Bilton was the Global Principal Investigator for CF301, and had full access to all the data in the study and final responsibility to submit for publication; P. Robinson was Australian Lead Regional Investigator for CF301; P. Cooper was an Investigator for CF301; C.G. Gallagher was Ireland Lead Regional Investigator for CF301; J. Kolbe was New Zealand Lead Regional Investigator for CF301; H. Fox approved the statistical plans and interpreted the data; A. Jaques was the CF301 protocol author and clinical study report author; and B. Charlton designed the CF301 study, approved the statistical plans, interpreted the data and was the sponsor's Responsible Medical Officer.

The authors wish to thank A. Solterbeck (Statistical Revelations, Melbourne, Australia) for her statistical analysis and expertise.

\section{REFERENCES}

1 Davies JC, Alton EW, Bush A. Cystic fibrosis. BMJ 2007; 335: 12551259.

2 Riordan JR, Rommens JM, Kerem B, et al. Identification of the cystic fibrosis gene: cloning and characterization of complementary DNA. Science 1989; 245: 1066-1073.

3 Ratjen FA. Cystic fibrosis: pathogenesis and future treatment strategies. Respir Care 2009; 54: 595-605.

4 Puchell E, Bajolet O, Abely M. Airway mucus in cystic fibrosis. Paediatr Respir Rev 2002; 3: 115-119.

5 Tarran R, Grubb BR, Parsons D, et al. The CF salt controversy: in vivo observations and therapeutic approaches. Mol Cell 2001; 8: 149-158.

6 Matsui H, Grubb BR, Tarran R, et al. Evidence for periciliary liquid layer depletion, not abnormal ion composition in the pathogenesis of cystic fibrosis airways disease. Cell 1998; 95: 1005-1015. 
7 Ramsey BW. Management of pulmonary disease in patients with cystic fibrosis. N Engl J Med 1996; 335: 179-188.

8 Lieu TA, Ray GT, Farmer G, et al. The cost of medical care for patients with cystic fibrosis in a health maintenance organization. Pediatrics 1999; 103: e72.

9 Zemanick ET, Harris JK, Conway S, et al. Measuring and improving respiratory outcomes in cystic fibrosis lung disease: opportunities and challenges to therapy. J Cyst Fibrosis 2010; 9: 1-16.

10 Flume PA, O'Sullivan BP, Robinson KA, et al. Cystic fibrosis pulmonary guidelines. Chronic medications for maintenance of lung health. Am J Respir Crit Care Med 2007; 176: 957-969.

11 Rowe RC, Sheskey PJ, Weller PJ, eds. Handbook of Pharmaceutical Excipients. Chicago, Pharmaceutical Press, 2003.

12 Daviskas E, Anderson SD, Brannan JD, et al. Inhalation of drypowder mannitol increases mucociliary clearance. Eur Respir J 1997; 10: 2449-2454.

13 Robinson M, Daviskas E, Eberl S, et al. The effect of inhaled mannitol on bronchial mucus clearance in cystic fibrosis patients: a pilot study. Eur Respir J 1999; 14: 678-685.

14 Anderson SD, Brannan J, Spring J, et al. A new method for bronchial-provocation testing in asthmatic subjects using a dry powder of mannitol. Am J Respir Crit Care Med 1997; 156: 758-765.

15 Brannan JD, Anderson SD, Perry CP, et al. The safety and efficacy of inhaled dry powder mannitol as a bronchial provocation test for airway hyperresponsiveness: a phase 3 comparison study with hypertonic (4.5\%) saline. Respir Res 2005; 6: 144.

16 Daviskas E, Anderson SD, Eberl S, et al. Inhalation of dry powder mannitol improves clearance of mucus in patients with bronchiectasis. Am J Respir Crit Care Med 1999; 159: 1843-1848.

17 Daviskas E, Anderson SD, Eberl S, et al. The 24-h effect of mannitol on the clearance of mucus in patients with bronchiectasis. Chest 2001; 119: 414-421.

18 Jaques AJ, Daviskas E, Turton JA, et al. Inhaled mannitol improves lung function in cystic fibrosis. Chest 2008; 133: 1388-1396.

19 Teper A, Jaques A, Charlton B. Inhaled mannitol in patients with cystic fibrosis: a randomised open-label dose response trial. J Cyst Fibros 2011; 10: 1-8.

20 Quittner AL, Buu A, Messer MA, et al. Development and validation of the Cystic Fibrosis Questionnaire in the United States: a health-related quality-of-life measure for cystic fibrosis. Chest 2005; 128: 2347-2354.
21 Fuchs HJ, Borowitz DS, Christiansen DH, et al. Effect of aerosolized recombinant human DNase on exacerbations of respiratory symptoms and on pulmonary function in patients with cystic fibrosis. N Engl J Med 1994; 331: 637-642.

22 Quittner AL, Modi AC, Wainwright C, et al. Determination of the minimal clinically important difference scores for the Cystic Fibrosis Questionnaire - Revised respiratory symptom scale in two populations of patients with cystic fibrosis and chronic Pseudomonas aeruginosa airway infection. Chest 2009; 135: 16101618.

23 Minasian CC, Wallis C, Metcalfe C, et al. Comparison of inhaled mannitol, daily rhDNase, and a combination of both in children with cystic fibrosis: a randomised trial. Thorax 2010; 65: 51-56.

24 Amadori A, Antonelli A, Balteri I, et al. Recurrent exacerbations affect FEV1 decline in adult patients with cystic fibrosis. Respir Med 2009; 103: 407-413.

25 Ziaian T, Sawyer MG, Reynolds KE, et al. Treatment burden and health-related quality of life of children with diabetes, cystic fibrosis and asthma. J Paediatr Child Health 2006; 42: 596-600.

26 Jones AP, Wallis C. Dornase alfa for cystic fibrosis. Cochrane Database Syst Rev 2010; 3: CD001127.

27 Elkins MR, Robinson M, Rose BR, et al. A controlled trial of longterm inhaled hypertonic saline in patients with cystic fibrosis. $N$ Engl J Med 2006; 354: 229-240.

28 Sawicki GS, Sellers DE, Robinson WM. High treatment burden in adults with cystic fibrosis: challenges to disease self-management. J Cyst Fibros 2009; 8: 91-96.

29 Modi AC, Marciel KK, Slater SK, et al. The influence of parental supervision on medical adherence in adolescents with cystic fibrosis: developmental shifts from pre to late adolescence. Child Health Care 2009; 37: 78-92.

30 Kesser KC, Geller DE. New aerosol delivery devices for cystic fibrosis. Respir Care 2009; 54: 754-767.

31 Flume PA, Mogayzel PJ, Robinson KA, et al. Cystic fibrosis pulmonary guidelines, pulmonary complications: hemoptysis and pneumothorax. Am J Respir Crit Care Med 2010; 182: 298-306.

32 Bartholdson SJ, Brown AR, Mewburn BR, et al. Plant host and sugar alcohol induced exopolysaccharide biosynthesis in the Burkholderia cepacia complex. Microbiology 2008; 154: 2513-2521.

33 Konstan MW, Flume PA, Kappler M, et al. Safety, efficacy and convenience of tobramycin inhalation powder in cystic fibrosis patients: the EAGER trial. J Cyst Fibros 2011; 10: 54-61. 